\title{
Research on Radio Fuze Ranging Method Based on Multi Carrier and Linear Frequency Modulation ${ }^{1}$
}

\author{
Jie Zhou, ${ }^{1, a}$, Quanli Ning ${ }^{2, b}$, Chunsheng $\mathrm{Hu}^{3, \mathrm{c}}$ \\ ${ }^{1,2,3}$ Army Officer Academy,Hefei,Anhui,230031,China \\ a962854399@qq.com, ${ }^{\text {b3025508944@qq.com, }{ }^{\text {c}} 842007264 @ q q . c o m}$
}

\begin{abstract}
Keywords: Multi carrier, Linear FM, Radio fuze distance.
Abstract. The nonlinear frequency modulation technology is applied to the fuze detection system, which makes the modulation signal in a period of a nonlinear change, so that the interference of the enemy is difficult to detect. Studies have shown that the fuze system has good speed resolution performance and the distance resolution, strong anti-jamming performance and the signal has thumbtack ambiguity function, in a modulation signal cycle can also provide non fuzzy distance and speed detection.
\end{abstract}

\section{Introduction}

With the development of modern warfare and its characteristics, it is required to meet the comprehensive requirements of the detection range, detection accuracy, range resolution, anti jamming and its economic performance. The modulation waveform of the radio fuze has important effect on improving the recognition ability of fuze target, anti jamming ability and reducing the ambiguity of measurement.

The linear frequency modulation continuous wave detection system has the advantages of low power, low probability of interception, high range resolution, and has been widely used in guidance and fuze technology. The basic principle is to detect the target distance by using the signal frequency between the transmitted signal and the target echo signal to reflect the target's distance information. But because of the ambiguity function of linear frequency modulation continuous wave fuze signal, the target can not get the exact velocity and range value of the target, and it is easy to be detected by the enemy.

In order to study the radio fuze detection system, it is a single carrier detection system. The target information contained in the system is limited, and the target echo signal is limited. According to the characteristics of multi carrier communication system based on multi carrier and linear FM modulation technology, a multi carrier radio fuze detection system is proposed. Compared with the single carrier radio fuze detection system, multi carrier and linear frequency modulation fuze can output more target information and electromagnetic environment information. These information are in the echo signal, so it is the key to adjust the parameters of the system. The orthogonal frequency division multiplexing (OFDM) detection technique can obtain good fixed distance and anti - jamming performance, but it is very sensitive to the leakage of antenna. With the signal processing method and the control method of multi carrier and linear frequency modulation complex modulation detection system, the target detection performance and anti jamming performance of multi carrier and linear frequency modulation compound modulation detection system can be directly determined.

\section{Time domain distance estimation}

OFDM signal after two times of mixing processing will be $\tau$ with the time delay:

$$
u(t)=s_{0}(t-\tau)=\sum_{i=0}^{N-1} d_{i} \exp \left[j 2 \pi \frac{i}{T}(t-\tau)\right]
$$

After sampling the signal, the discrete signal is obtained, which is expressed as follows: 


$$
u(n)=\sum_{i=0}^{N-1} d_{i} \exp \left[j 2 \pi \frac{i}{T}(n-m)\right]
$$

The sampling interval in the upper formula is $t_{s}=T / N, \mathrm{~m}=\left[\tau / t_{s}\right]$ represents delay sampling point. In the time domain discrete correlation processing, the output value of the sample points in the first $\mathrm{m}$ point of the maximum value of the model, corresponding to the sampling time $\mathrm{mt}_{\mathrm{s}}$ as the starting position of the FFT window, that is the coarse estimation delay is $\mathrm{mt}_{\mathrm{s}}$. The expression of the correlation function $R_{s s}\left(\tau_{n}\right)$ is as follows:

$$
\left|R_{s s}\left(\tau_{n}\right)\right|=\left.\left|T \cdot \sum_{i=0}^{N-1}\right| d_{i}\right|^{2} \exp \left(j 2 \pi \frac{i}{T} \tau_{n}\right) \mid=\frac{N \cdot T \cdot \sin c\left[N \frac{\tau_{n}}{T}\right]}{\sin c\left[\frac{\tau_{n}}{T}\right]}
$$

The self model correlation values in the zero value of the maximum graphics to approximate the sinc ( $\mathrm{x}$ ) and sequence code length $\mathrm{n}$ the longer the width of the main lobe narrower, the higher range resolution, the same sequence code length, single carrier autocorrelation main lobe width is $18.79 \mathrm{~dB}$ based on OFDM multi carrier $\mathrm{n}$ times that of the main lobe width and CAZAC sequence column modulus main to side lobe ratio, as shown in Figure 1 . The relative ranging accuracy is $t_{s}$, and the maximum error is $c \cdot t_{s} / 2$, so when the distance measurement system and the target distance is less than $c \cdot t_{s} / 2$. Because of the very narrow main lobe and high side lobe ratio based on the optimized sequence OFDM, if the sampling and interpolation techniques are used, the high noise environment, measuring distance and precision are not very high, the relative ranging can meet the requirements of detection performance.

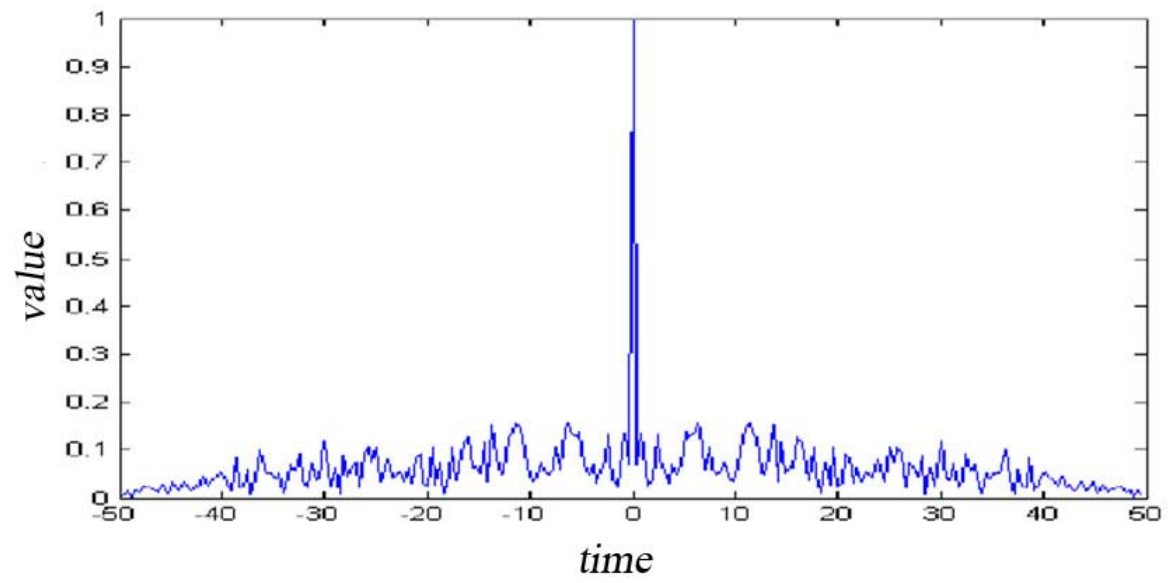

Figure 1 auto correlation value of time domain signal

\section{Distance estimation in frequency domain}

After the estimation of the time domain, the receiver can determine the OFDM sign of the fuze detector. The OFDM signal can be transformed to the frequency domain by FFT transform. The phase difference between the fuze and target is extracted.

OFDM signal has been known to:

$$
s_{0}(t)=\sum_{i=0}^{N-1} d_{i} \exp \left(j 2 \pi \frac{i}{T} t\right)
$$

In the formula, $d_{i}=\exp \left(j \phi_{i}\right)$, the phase difference between adjacent sub carriers in frequency domain is $\Delta \phi_{t}=\phi_{i+1}-\phi_{i}$, as reference phase, $\phi_{i}$ is data phase for sub carrier 。

Assuming that the maximum correlation value of the signal corresponding to the theoretical delay is $\tau_{0}$, and not in the integer sampling point, the time domain estimation delay is $\mathrm{mt}_{\mathrm{s}}$, known as the fractional delay $\tau^{\prime}=\tau-m t_{s}$, the discrete signal after A/D conversion by FFT transform, the 
fractional delay causes the phase rotation, compared with the original data, FFT output data $\hat{d}_{i}$ in the original data $d_{i}$ on the basis of the rotation phase, that is:

$$
\begin{gathered}
\hat{d}_{i}=d_{i} e^{-j 2 \pi f_{i} \tau^{\prime}}=e^{-j\left(2 \pi f_{i} \tau^{\prime}-\phi_{i}\right)}, 0 \leq i \leq N-1 \\
\hat{d}_{i} d_{i}^{*}=d_{i} d_{i}^{*} e^{-j 2 \pi f_{i} \tau^{\prime}}=\left|d_{i}\right|^{2} e^{-j 2 \pi f_{i} \tau^{\prime}}, 0 \leq i \leq N-1
\end{gathered}
$$

Assuming that the frequency difference between adjacent sub carriers is (6), the phase difference between adjacent sub carriers is seen:

$$
\Delta \phi_{t}^{\prime}=-2 \pi \Delta f \tau^{\prime}+\Delta \phi_{i}, 1 \leq i \leq \frac{N}{2}
$$

Compared with the reference phase, that is $\Delta \phi_{t}=\phi_{i+1}-\phi_{i}$, is obtained from the formula (7):

$$
\begin{gathered}
\Delta \theta_{i}=\Delta \phi_{i}^{\prime}-\Delta \phi_{i}=2 \pi \Delta f \tau_{i}^{\prime} \quad 1 \leq i \leq N / 2 \\
\tau_{i}^{\prime}=\left|\frac{\Delta \theta_{i}}{2 \pi \Delta f}\right| \quad 1 \leq i \leq \frac{N}{2}
\end{gathered}
$$

$\tau_{i}^{\prime}$ is obtained by phase difference between adjacent sub carriers in L. On the arithmetic average of $\tau_{i}^{\prime}$ as the estimation of time delay of the $\tau^{\prime}$.

$$
\tau^{\prime}=\frac{2}{N} \sum_{i=1}^{N / 2} \tau_{i}^{\prime}=\left|\frac{2}{N} \sum_{i=1}^{N / 2} \frac{\Delta \theta_{i}}{2 \pi \Delta f}\right|=\frac{|\overline{\Delta \theta}|}{2 \pi \Delta f}
$$

$\overline{\Delta \theta}$ is the arithmetic mean of $\Delta \theta_{i}$, by the signal detection and estimation theory, the estimation is asymptotically unbiased and the real time delay is estimated:

$$
\tau=m t_{s}+\tau^{\prime}
$$

Then target distance is:

$$
R=\frac{c \tau}{2}=\frac{c|\overline{\Delta \theta}|}{4 \pi \Delta f}+\frac{c m t_{s}}{2}
$$

Phase difference between the sub carrier and reference phase difference can be interval of a plurality of sub carriers, $\Delta \theta$ can arbitrarily choose anyone of $\{2 \pi \Delta f \tau, 2 \pi \cdot 2 \Delta f \tau, \cdots 2 \pi \cdot(N-1) \Delta f \tau\}$ after phase comparison, in order to reduce the phase noise of frequency estimation, usually should choose the sub carrier spacing as large as possible.

\section{Ranging performance analysis}

Range resolution and maximum distance are the key indicators to measure the performance of fuze ranging. Because of the periodicity of the correlation function, the range of each correlation value is corresponding to the number of distance, and if it is not correct, it can not be achieved accurately. When the signal from the fuze launch end to the target round-trip delay just for an OFDM signal period $\mathrm{T}$, the distance between missile and target for maximum unambiguous range.

$$
R_{\max }=\frac{c T}{2}
$$

Range resolution is the ability to distinguish between the minimum distance of the fuze, that is $R_{\min }$. OFDM and linear frequency modulation fuze detection system uses the method of time and frequency processing, usually in the practical application, it can be used to meet the need of fuze, the distance resolution can be expressed as: 


$$
R_{\min }=\frac{c T_{s}}{2}
$$

Estimation based on time domain using frequency domain estimation, can calculate the fractional delay of less than Ts is $\tau_{\min }^{\prime}$, can estimate the corresponding decimal distance, further enhance the distance resolving power of fuze.

\section{References}

[1] Zhao Huichang. Design principle and method of radio fuze. First ed. Beijing: National Defense Industry Press, 2012

[2] Song Shihe, Cui Zhanzhong, Xu Lixin. Principle of proximity fuze. Second ed. Beijing: Beijing Institute of Technology press, 2005

[3] Xu Jianzhong. Wireless sensor system. First ed. Nanjing: Nanjing University of Science and Technology press, 2005

[4] Merrill Lskolnik. Radar manual. Third ed. Beijing: Electronic Industry Press, 2010

[5] Geng Qi. Research on Fuze Signal Processing Technology of FM Doppler. Nanjing: Nanjing University of Science and Technology, 2008 